\title{
ABORDAGEM CIRURGICA INTRA E EXTRADURAL DAS MENINGOENCEFALOCELES DO ANDAR ANTERIOR
}

\author{
J. PINDARO P. PLESE * \\ MARIO AUGUSTO TARICCO * \\ GILBERTO MACHADO DE ALMEIDA **
}

Várias abordagens cirúrgicas tem sido relatadas para correção de meningoencefaloceles do andar anterior. Todas visam à ressecção completa da malformação e a adequada reconstituição facial.

Nosso objetivo é descrever técnica cirúrgica que usa a via intra e extradural da malformação e apresentar os resultados obtidos, com este procedimento, em 5 pacientes.

\section{MATERIAL E METODOS}

A casuistica s constituída de 5 pacientes internados entre 1975 a 1977. A tabela 1 fornece a identificação dos pacientes, os resultados obtidos e o tempo de seguimento pós-operatório.

Em todos os casos, o único dado de história era a tumoração frontonasal desde o nascimento. Uma criança (caso 4) foi submetiła a cirurgia extracraniana anterior e houve subsequente crescimento exagerado da tumoração. $O$ exame neurológico não evidenciou anormalidades.

Os meios paraclínicos utilizados para confirmação diagnóstica foram: radiografias simples de crânio, eletrencefalograma e cisternocintilografia com soro albumina marcada com 131I (RHISA). Nos casos 2, 3, 4, a RHISA não fol usada.

Todos os pacientes foram submetidos a craniotomia bifrontal, abordando-se a malformaçåo por via intra e extra-dural. Após ressecção da encefalocele foi feito cuidadoso fechamento da dura-mater, colocando-se enxerto ósseo homólogo na falha craniana do andar anterior.

Quanto aos resultados, a evoluçăo foi satisfatória, com tempo de permanencia hospitalar pós-operatório de 7 dias. Os pacientes 1,2 e 3 foram submetidos a cirurgia plástica com bons resultados cosméticos após três meses da cirurgia intracraniana. Nâo houve ocorrência de fístula do líquido cefalorraquidiano (LCR), infecçóes ou convulsōes no pós-operatório.

Técnica cirurgica - Paciente em decúbito dorsal horizontal sob anestesia geral combinada (endovenosa e inalatória), é submetido a craniotomia bifrontal osteoplástica rasante ao rebondo orbitário superior, após incisăo bicoronariana dos planos superficiais (Fig. 1 A). No polo frontal direito, dois fios de sutura (mononylon 4.0) são

Trabalho da Clínica Neurológica da Faculdade de Medicnia da Universidade de Săo Paulo (Prof. H. M. Canelas): * Assistente Auxiliar de Ensino:** Residente de Neurocirurgia;** Assistente-Docente, Chefe do Setor de Neurocirurgia. 


\begin{tabular}{cllcll}
\hline Caso & Nome & Idade & Sexo & Cor & $\begin{array}{c}\text { Seguimento } \\
\text { pós-operatório }\end{array}$ \\
\hline 1 & CBA & 8 meses & feminino & preta & 3 anos \\
2 & AMS & 2 anos & masculino & branca & 1 ano e 1 mês \\
3 & TCO & 17 anos & feminino & parda & 4 meses \\
4 & PTBS & 9 meses & masculino & branca & 3 meses \\
5 & VO & 1 ano & feminino & preta & 1 mês \\
\hline
\end{tabular}

Tabela 1 - Dados de identificaşão dos 5 pacientes e o periodo de seguimento posopcratorio.

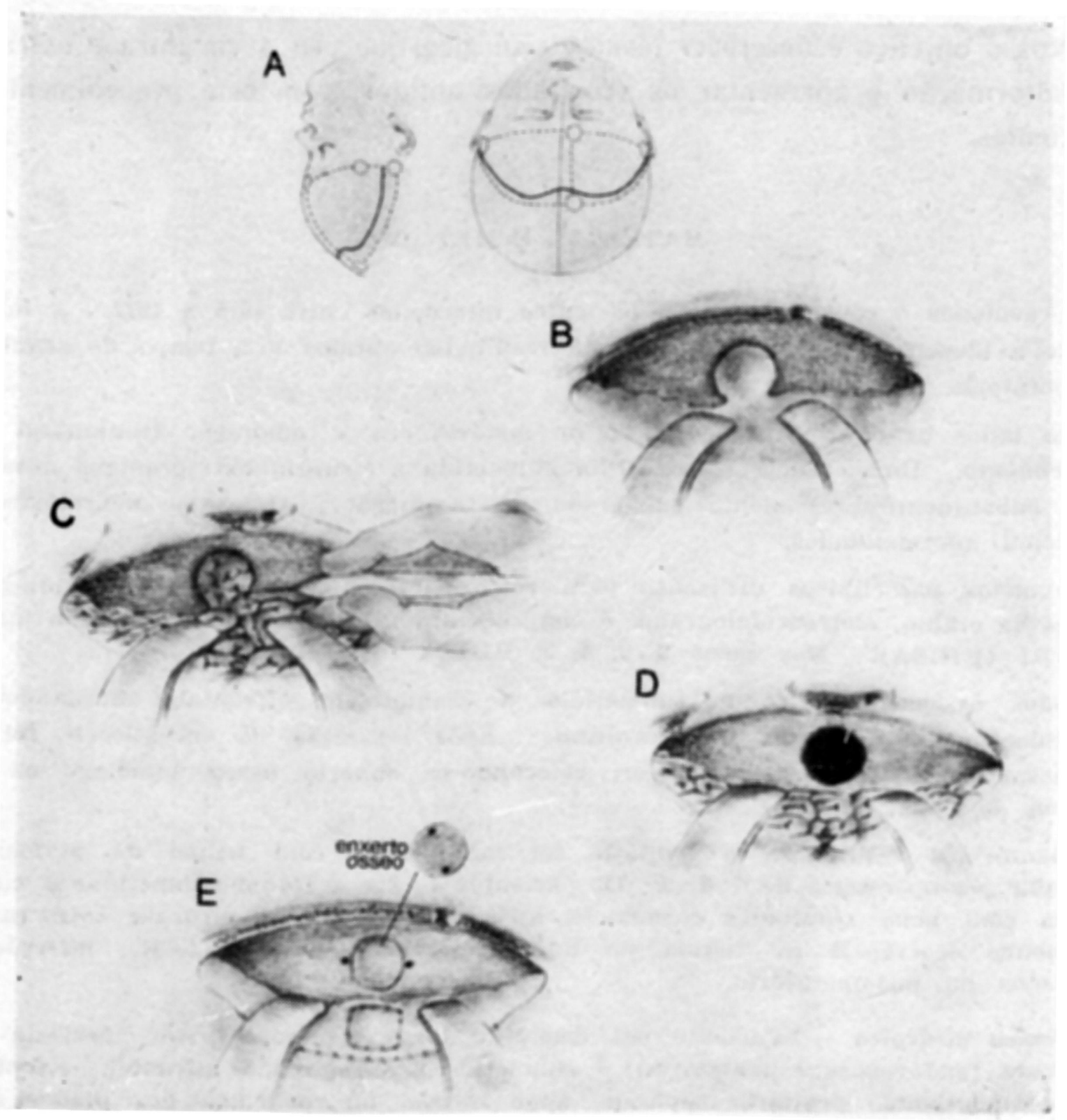

Fig. 1 - Em $A$, incisăo do couro cabeludo $e$ locais dos orificios de trepanacąe 68sea; em $B$, dissecço da malformaço por via cxtra-dural delimitando-se suas bordas; em $C$, dissecça da meningoencefalocele por via intra-dural e ressecço da mesma; em $D$, exposigão do orificio por onde ha protrusao da meningoencefalocele; em $E$; fechamento da dura-mater e colocacto de enxerto bs8eo. 
passados através da dura-mater, separados de três a quatro centímetros, sendo feito pequeno pertuito na dura-mater e aracnóide, o suficiente para que haja saida de LCR. Por via extra-dural, afastando-se os lobos frontais, procede-se a abordagem da meningocele e dissecç̊o cuidadosa de suas bordas anterior, laterais e posterior (Fig. 1.B) tomando-se cuidado para năo romper a meninge. A seguir é feita abertura da dura-mater na porçăo anterior dos lobos frontais, ligadura de seio sagital superior, seç̧̆م do mesmo e da foice inter-hemisférica. Com o retrator de Yasargil afastam-se os lobos frontais, protegendo-os com gelfoam e disseca-se o colo da encefalocele; com o auxílio do coagulador bipolar a mesma é ressecada (Fig. 1 C). Protege-se a porçá cruenta com celulose oxidada regenerada. Usa-se cureta maleável para retirar $b$ conteúdo herniado. Por via extra-dural resseca-se a dura-mater invaginada e a que forra as bordas da falha 6ssea (Fig. I D). A falha dural é reparada com enxerto de periósteo. A sutura da parte posterior é feita por via intra-dural e as bordas laterais e anterior sấ suturadas por via extra-dural com pontos separados (mononylon 4.0). Coloca-se fragmento 6sseo retirado do "flap" da craniotomia na falha do andar anterior (Fig. $1 \mathrm{~F}$ ), e apōe-se sobre este perísteo 0 músculo. A dura-mater é suturada com pontos separados (mononylon 4.0). Procede-se ao fechamento clássico da craniotomia. Quando a falha óssea do andar anterior é extensa, realiza-se fixaçáo do enxerto ósseo.

\section{DISCUSSxO}

Um dos primeiros tratamentos cirúrgicos desta malformação consistia na abordagem do processo por via extracraniana 4. Vários autores ${ }^{3,5,6,6,70}$ evidenciaram os inconvenientes dessa via de acesso, ressaltando os péssimos resultados estéticos, alta incidência de infecção de fístulas do LCR e a ressecção incompleta da malformação.

Matson ${ }^{6}$ estabeleceu o tratamento cirúrgico por via intracraniana e intradural, com o qual obteve melhores resultados pós-operatórios, dados estes confirmados por Dodge e col.5 e Anderson ${ }^{3}$. A via intracraniana extra-dural tem sido usada por muitos $2,8, \mathbf{\theta}, 11$ justificada por ser possivel uma melhor sutura da dura-mater, evitando as fístulas do LCR. Adrianjakovo ${ }^{1}$, em 1968, afirma que bons resultados independem da via usada (intra ou extra-dural), sendo diretamente relacionadas à cuidadosa técnica cirúrgica. Quanto ao fechamento da falha óssea do andar anterior, têm sido usados enxertos ósseos autólogos e homólogos, placas de vitalio, tântalo e aço, metil-metacrilato e polietileno.

A técnica cirúrgica proposta neste trabalho, apresenta as seguintes vantagens em relação às técnicas mencionadas anteriormente: pela via extra-dural tem-se melhor visualização do saco dural e seu relacionamento com os planos ósseos, facilitando a sutura da dura-mater e permitindo, inclusive, a realização de osteossintese da falha óssea, se necessário; a via intra-dural fornece melhor campo cirúrgico para a vizualização da encefalocele, e exame da impermeabilidade da sutura da dura-mater; com retirada de bom volume de LCR e boa proteção dos lobos frontais, com gelfoam, não ocorre lesão cortical; o uso de enxerto homólogo na falha óssea apresenta vantagens sobre o emprego de corpos estranhos.

Portanto a via intracraniana, com abordagem intra-dural e extra-dural combinadas, nos proporciona as vantagens das vias intra e extra-durais, com ótimos resultados pós-operatórios, como se verificou nos 5 casos descritos neste trabalho. 


\section{RESUMO}

E descrita a técnica de abordagem das meningoencefaloceles do andar anterior, por via intra e extra-dural. Os resultados obtidos em cinco pacientes foram considerados bons.

\section{SUMMARY}

Surgical treatment of ethmoidal encephalomeningoceles: intra and extra dural aproach.

The intra and extra dural aproach for the treatment of ethmoidal encephalomeningoceles is described. A comparation with other surgical aproaches is made and good results obtalned in 5 cases are reported.

\section{REFERENCTAS}

1. ADRIANJAKOVO, J. \& PIALOUX, P. - Aperçus sur les meningoenphaloceles anterieurs: a propos de 9 cas. Ann. Otolaring. 85-867-696, 1968.

2. AISEN, J.; PEREIRA, W. C.; ANDRADE, A. F. \& PSILAKIS, J. M. - Anterior floor meningoencephaloceles surgical treatment. Arq. Neuro-Psiquiat. (Săo Paulo). $34: 105-115,1976$.

3. ANDERSON, F. M. - Intranasal (sphenopharyngeal) encephalocele: a report of a case with intracranial repair and a review of the subject. Arch. Otolaring. 46: 644, 1947.

4. DANDY, W. E. - The Brain. Section VI pág. 243-251. Hoeber Medical Divison. New York, 1969.

5. DODGE, H. W. Jr.; LOVE, J. G. \& KERNOHAN J. W. - Intranasal encephalomeningoceles associated with cranium bifidum. Arch. surg. 79:75. 1959.

6. MATSON, DONALD D. - Neurosurgery of Infancy and Childhood. Part I, Chapter 2:61-75 Charles C. Thomas Publisher, Springfield, 2nd Edition, 1969.

7. McCUILLCODDY, O. B. - Encephalomeningoceles in the nasal cavities. Ann. Otol. Rhinol. Lar. 51:516, 1942.

8. MOORE, P. M. - Intranasal encephalomeningocele: report of a case. Trans. Amer. Lar. Rhinol. Otol. Soc., 50th meeting, Cleveland, 1952, pp 481-502.

9. OBLU, N.; SANDELESCU, G. \& RUSU, M. - Meningoencephalocelul anterior al bazei craniului. Oto-rino-laringologie 14:147, 1969.

10. TESSIER, P.; CUIT., G.; ROUGERIER, J.; DELBET, P. \& PASTORIZA, J. Osteotomies cranio-naso-orbito-faciales. Hipertelorisme. Ann. Chir. plast. 12:103, 1967.

11. WALKER, E.; MOORE, W. W. \& SIMPSON, J. R. - Intranasal encephaloceles: Survey of problem with recommendations for reducing mortality. Arch. Otolaring 55:182, 1952. 